\title{
EFEITO DE HERBICIDAS RESIDUAIS, APLIC ADOS POR VÁRIOS ANOS \\ CONSECUTIVOS, NA DISTRIBUIÇÃO DO SISTEMA RADICULAR \\ DA LARANJEIR A NATAL (Citrus sinensis.(L.) OSBECK)
}

\section{R VICTÓRIA FILHO \\ J.C. DURIGAN ${ }^{2}$, I. ANDRIOLI ${ }^{2}$ \& E.M. GUSMÃ O ${ }^{3}$}

${ }^{1}$ Professor Adjunto da Escola Superior de Agricultura "Luiz de Queiroz"-ESALQ-USPDepto. de Horticultura, Piracicaba, SP.

${ }^{2}$ Professores-Doutores da Fac. de Ciências Agrárias e Veterinárias, Jaboticabal - UNESP - Depto. De Defesa Fitossanitária Jaboticabal, Jaboti SP.

${ }^{3}$ Acadêmico da FCAVJ-UNESP.

\section{RESUMO}

O presente trabalho foi desenvolvido com o objeti vo de verificar a influência do uso contínuo de vários herbicidas residuais, sobre a distribuição das radicelas de laranjeiras Natal (Citrus sinensis (L.) Osbeck) enxertadas sobre limão Cravo (Cithus limonia Osbeck) e plantadas em janeiro de 1970.

$\mathrm{O}$ experimento foi instalado no pomar de produção da Faculdade de Ciências Agrárias e Veterinárias do Campus de Jaboticabal (UNESP), em Latossol Roxo distrófico, com 1,6\% de matéria orgânica e $51 \%$ de argila.

O delineamento experimental utilizado foi o de blocos ao acaso, dentro de um esquema fatorial $11 \times 3 \times 2 \times 2$, com três repetições. Os tratamentos utilizados, com as respectivas doses em $\mathrm{kg}$ do i.a/ha foram: fluometuron a 4,2 ; simazíne a 4,8; atrazine a 4,8; bromacil a 3,2 ; bromacil $(40 \%)+$ diuron $(40 \%)$ a 4,8 ; bromacil $(53,3 \%)+$ diuron $(27,6 \%)$ a 4,8 ; terbacil a 3,2; oxadiazon a 1,5; dichlobenil a 3,0 e 6,0; além de uma testemu nha capinada.

$\mathrm{O}$ estudo das radicelas foi feito pelo "método do trado", tendo sido tomadas amostras de solo contendo radicelas, nas distâncias de 80, 160 e $240 \mathrm{~cm}$ do tronco, nas camadas de 0 a $15 \mathrm{~cm} \mathrm{e} 15$ a $30 \mathrm{~cm}$ de profundidade e em duas direções distintas (entrelinhas e entreplantas).

Os resultados mostraram que as radicelas localizam-se superficialmente, com $70 \%$ delas nos primeiros $15 \mathrm{~cm}$, do total encontrado na camada superficial de 0 a $30 \mathrm{~cm}$ de profundidade do solo. Cerca de $75 \%$ das radicelas situam-se até a distância de $160 \mathrm{~cm}$ do tronco. Na camada mais superficial do solo (0 a $15 \mathrm{~cm}$ ), a quantidade de radicelas diminuiu de maneira acentuada, à medida que se afastou do tronco. Na camada de 15 a 30 cm de profundidade, ocorreu uma distri- 
buição horizontal mais uniforme, comparada com a da camada de 0 a $15 \mathrm{~cm}$.

As radicelas das plantas do cultivar Natal distribuiram-se uniformemente, tanto na direção das plantas da linha vizinha, quanto nas das plantas de uma mesma linha, para o espaçamento de 7,0 m x 7,2 m.

Não se detectaram diferenças estatisticamente significativas nas quantidades de radicelas entre as parcelas dos vários tratamentos com herbicidas, excluindo-se desta forma, qualquer efeito negativo de tais produtos químicos sobre o sis tema radicular das laranjeiras.

PALA VRAS -CHAVE: he rbicid as, sistema radicular, laranja Natal.

\section{SUMMARY}

\section{EFFECTS OF RESIDUAL HERBICIDES, APPLYED FOR SEVERAL YEARS ONTHE ROOT SYSTEM DISTRIBUTIONOF \\ 'NATAL' ORANGE (Citrus sinensis L. OSBECK).}

This experiment was carried out at the orchard of Faculdade de Ciências Agrárias e Veterinárias - Campus de Jaboticabal UNESP, with the objective to verify the influence of the continuous use of several residual herbicides on radicals distribution of 'Natal' orange (Citrus sinensis (L.) Osbeck) grafted on 'Cravo' lemon (Citrus Limonia Osbeck); they were planted on january, 1970, in a soil classified as Orthox with $1,6 \%$ of organic matter.

The test had a randomized blocks experimental design in a $113 \times 2 \times 2$ factorial design with three eplications. Treatments with their respective doses ( $\mathrm{kg}$ a.i/ha) were: fluo meturon at 4.2 ; sima zine at 4.8 ; atra zine at 4.8 ; bromacil at 3.2 ; bromacil $(40 \%)+$ diuron $(40 \%)$ at 4.8 ; $\operatorname{bromacil}(53.3 \%)+$ diuron $(26.7 \%)$ at 4.8 ; terbacil at 3.2 ; oxadiazon at 1.5; dichlobe - nil at 3.0 and 6.0, and a control with cleaning.

The study of radicels was done by the "anger method" by taking soil samples containing radicels at the distances of 80 , 160 and $240 \mathrm{~cm}$ from the trunk at layers of 0-15 cm and 15-30 cm of depth and in two distinct diretions (between lines and between plants).

Results showed that radicels are superficially located, being $70 \%$ of them in the layer of $0-15 \mathrm{~cm}$ of soil depth and the total amount of them in the layer of $030 \mathrm{~cm}$ of depth. About $75 \%$ of the radicels are located till adistance of $160 \mathrm{~cm}$ from the trunk. In the upper soil layer (0$15-\mathrm{ccm})$, the amount of radicels markedly decreases as increases the distance from the trunk. In the layer of $15-30 \mathrm{~cm}$, there occurred an horizontal and more uniform distribution of radicels when compared with the $0-15 \mathrm{~cm}$ layer.

No differences were found for the radicels amount among the different treatments showing that these herbicides have no negative effects on the root system of 'Natal' orange.

KEYW ORDS: herbicides, root system, 'Natal" orange.

\section{INTRO DUÇÃO}

Para o emprego de certas praticas culturais, relacionadas com $\mathrm{O}$ controle de plantas daninhas e adu bação, e de fundamental importân - 
cia o conhe cimento do sistema radic ular das plantas cítricas, de for ma a evitar danos irreversí veis que comprometam a vida útil do pomar ou que levem ao não aproveita mento do fertilizante aplicado.

Dentre as praticas culturais utilizadas pelos citricultores, o controle químico do mato vem recebendo certa atenção principalmente devido a escassez e alto custo da mão-de-obra no meio rural, dentro das principais regiões produtoras dos cítricos. Apesar disso o uso de herbicidas ainda é pequeno, comparado com outros pauses produtores.

$\mathrm{O}$ uso de herbicidas como alternativa para o controle de plantas daninhas tem sido considerado, em função de algumas vantagens que apresenta, tais como, custo menor, eficacia e rapidez operacional. Varios herbicidas disponíveis no mercado apresentam boa seletividade para as plantas cítricas e são utilizados em grande parte das áreas produtoras do mundo, como nos EUA (8, 10, 15, 16), na Italia (17), em Israel (4), no Japão (14) e na África do Sul (6).

No Brasil, o uso de herbicidas em pomares cítricos comerciais é relativamente recente e se mantém em pequena escala, principalmente para os herbicidas de solo (residuais) tendo como uma das principais razões a falta de informações sobre os possíveis efeitos nas plantas cítricas.

Durigan (2) afirma que, no Brasil, apenas as grandes empresas agrícolas adotamo controle químico de plantas daninhas nos seus pomares, em virtude da necessidade de um perfe ito atendimen to a fatores de natureza técnica, econômica e cultural, dando possibilidade para que esta prática venha a ser realizada de manei- ra racional. Relat a também que, para certas regiões do Estado de São Paulo, a falta de mão-de-obra, em determinadas épocas do ano devido a concorrên cia com outras culturas, vem motivando alguns citricultores ao emprego do controle químico. Segundo Caetano (1), somente 5 a $10 \%$ da área plantada era tratada com herbicidas e explica que isto se deve, principalmente, à falta de mão-de-obra capacitada.

A partir do momento em que a molécula de um certo herbicida atinge o solo, ela começa a exercer sua ação fitotóxica e ao mesmo tempo a sofrer aço de microorganismos, da luz solar e da tempe ratura, além das reações químicas (hidrólise, oxidação, redução, etc). O movimento, a atividade e a persistên cia dos herbicidas no solo afetam sua eficácia e sua seletividade em pomares cítricos (9). A toxicidade dos herbicidas as plantas cítricas, depende do movimento de água no solo, da solubilidade do produto e das característica s do solo. Como cons eqüência, o equilíbrio entre a quantidade do herbicida em solução e adsorvida aos colóides do solo, determinam a extensão do controle e/ou intoxicação das plantas de interesse econômico.

Kimball et al. (1950), citados por Jordan \& Day (8), estudaram a influência do manejo das plantas daninhas, utilizando-se herbicidas e gradagens, sobre a quantidade de radicelas, em laranjeiras da California. Mostraram que na ausência de gradagens, $71 \%$ das radicelas estavam nos $70 \mathrm{~cm}$ superiores do perfil do solo sendo que apenas $50 \%$ delas foram encontradas em solo gradado. Concluíram que o controle químico do ma- 
to, feito racionalmente, além de não prejudicar o desenvolvimento, pode favorecer o crescimento de radicelas, principalmente nas camadas mais superficiais do solo. Por outro lado, Moreira (13) encontrou redução de radicelas na faix a tratada, quando testou capinas freqüentes e emprego de herbicidas (terbacil, bromacil, paraquat, glyphosate e MSMA) em laranjeira 'fera'.

Este trabalho de pesquisa teve por obje tivo, obter maiores infor mações sobre o desenvolvimento do sistema radicular de plantas cítricas submetidas à aplicações de herbicidas residuais por vários anos consecutivos. Procurou-se analisar a influência de vários herbicidas, sobre a distribuição horizontal e vertical do sistema radicular de laranjeiras 'Natal'.

\section{MATERIAL E MÉTODOS}

As amostras do siste ma radi cular foram retiradas em experimento instalado no pomar da Faculdade de Ciência Agrárias e Veterinárias Campus de Jaboticabal UNESP. A área experimental se acha localizad a em um Latossol Roxo, com 1,6\% de matéria orgânica; $31,4 \%$ de areia; $16,8 \%$ de silte; $51,8 \%$ de argila e uma densidade aparente igual a 1,26 g.cm ${ }^{-3}$, segundo dados obtidos por Victória Filho et al. (18).

As laranjeiras, plantadas em janeiro de 1970, foram obtidas de clone nucelar. A copa era cultivar Natal (Citrus sinensis L. Os-beck) enxertado em limoeiro 'Cravo' (Citrus limonia Osbeck) .

O deline amento utilizado foi o de blocos ao acaso, dentro de um esquema fatorial $11 \times 3 \times 2 \times 2$, com três repetições. Dessa forma os tratamentos constaram de 10 herbi- cidas aplicados ao solo, em préemergência das plantas daninhas e uma testemunha capinada. Para cada tratamento, foram coletadas amostras de solo contendo radicelas, em três distâncias do tronco $(80,160$ e $240 \mathrm{~cm}$ ), nas camadas de 0 a 15 e 15 a 30 $\mathrm{cm}$ de profundidade, em duas direções distintas (entrelinhas e entreplantas). Cada parcela era constituí-da por duas plantas, espaçadas de 7,2 m x 7,0 m, totalizando $100,8 \mathrm{~m}^{2}$.

Os tratamentos, contendo os herbicidas utilizados e as suas respectivas doses e formu lações, estão especificadas no Quadro 1. As aplicações iniciaram-se no ano de 1974 e se repetiram por nove anos consecutivos de acordo com Victória Filho et al. (18).

A coleta de amostras contendo raízes, efetuou-se no 19 semestre de 1924, seguindo a metodologia utilizada por Montenegro (12) e Moreira (13), com modificação apenas no diâmetro do trado para 7,5 cm. Foi estudado o sistema radicular de uma arvore de cada parcela, no total de 33 arvores. A arvore escolhida em cada parcela era sempre a segunda, caminhando-se no sentido nordeste para sudoeste.

Para obtenção de um volume de solo contendo raízes suficientes para uma avaliação segura do peso de radicelas, foram neces sárias duas amostras em cada distância, que posteriormente foram reunidas numa única, representativas das diferentes distâncias do tronco, dentro de cada profundidade. Dess a forma, cada amos tra composta possuía o volume de dois cilindros de $7,5 \mathrm{~cm}$ de diâmetro e $15 \mathrm{~cm}$ de altura tota lizan do $1.326,36 \mathrm{~cm}^{3}$.

O método adotado prevê o estu do do sistema radicular, utilizan do o 
Quadro 1. Tratamentos testados, apresentando os herbicidas com seus respectivos nomes comercial e comum (i.a), além das doses e formulações.

\begin{tabular}{|c|c|c|c|c|c|}
\hline \multirow{2}{*}{ No } & \multicolumn{2}{|c|}{ Herbicida } & \multicolumn{2}{|l|}{ Dose/ha } & \multirow{2}{*}{ Formulação } \\
\hline & Nome comercial & $\begin{array}{l}\text { Nome } \\
\text { comum }\end{array}$ & $\begin{array}{l}\text { p.c. } \\
\mathrm{kg} \text { ou 1) }\end{array}$ & $\begin{array}{l}\text { i.a. } \\
(\mathrm{kg})\end{array}$ & \\
\hline 1. & Testemunha capinada & & - & - & - \\
\hline 2. & Cotoran & fluometuron & 5 & 4,2 & PM $80 \%$ \\
\hline 3. & Gesatop & simazine & 6 & 4,8 & PM $80 \%$ \\
\hline 4. & Gesaprim & atrazine & 6 & 4,8 & PM $80 \%$ \\
\hline & Hyvar $\mathrm{X}$ & bromaci 1 & 4 & 3,2 & PM $80 \%$ \\
\hline & Krovar I & bromacil $(40 \%)+$ diuron $(40 \%)$ & 6 & 4,8 & PM $80 \%$ \\
\hline 7. & Krovar II & bromacil $(53,3 \%)+$ diuron $(26,7 \%)$ & 6 & 4,8 & PM $80 \%$ \\
\hline & Sinbar & terbacil & 4 & 3,2 & PM $80 \%$ \\
\hline 9. & Ronstar & oxadiazon & 6 & 1,5 & CE $250 \mathrm{~g} / 1$ \\
\hline 10. & Casoron & dichlobenil & 60 & 3,0 & GR $5 \%$ \\
\hline 11. & Casoron & dichlobenil & 20 & 6,0 & GR $5 \%$ \\
\hline
\end{tabular}

$\begin{array}{ll}\text { i.a - ingrediente ativo; } & \text { PM - pó molhável } \\ \text { p.c - produto comercial } & \text { GE - concentrado emulsionável } \\ & \text { GR - granulado }\end{array}$


tro igual ou menor do que $1,5 \mathrm{~mm}$, conforme critério proposto por Ford (3), que vem sendo adotado por diversos outros aut ores, con forme Moreir a (13).

Com os dados obtidos da pesage m das radicelas secas em estufa (60$70^{\circ} \mathrm{C}$ ), for am pos síveis as realizações das análises estatísticas bem como os cálculos das porcentagens existentes em cada dis tância, nas diferentes profundidades e dir eções.

\section{RESULTADOS E DISCUSSÃO}

Os resultados obtidos, referentes aos pesos médios (cg) das radicelas nas amostras coletadas à diferentes distâncias do tronco, profundidades do solo e direções, encontram-se no quadro 2.

No Quadro 3 esta a análise de variância dos pesos de radicelas, para os diferentes fatores avaliados e suas interações. A analise estatística dos resultados evidenciou uma variação significativa ao nível de $1 \%$ de probabilidade para a distancia da amostragem a partir do tronco e profundidade de coleta das amostras no solo. Dessa forma, próximo ao tronco, as raízes mais velhas aparesentam maior numero de radicelas e à medida em que se distancia, ocorre uma diminuição sensível. Da mesma forma, as radicelas diminuem à medida em que aumenta a profundidade do solo. Este fato, até certo ponto já era esperado e decorre da biologia das dicotiledôneas, que apresentam sistema radicular do tipo pivotante, classe botânica na qual estão inseridas as plantas cítricas.

Não foram constatadas dife renças signific ativas proporcion adas pelos diversos herbicidas, na dis - tribuição do sistema radicular das plantas. Desta forma, fica caracterizada a inocuidade de tal método de controle das plantas daninhas, pois as doses testadas no presente trabalho estio muito próximas do limite superior de indicação para o solo em que se encontra instalado o pomar. Mesmo o herbicida de maior solubilidade, como é o caso do bromacil, aplicado isoladamente, não proporcionou efeitos maléficos na quantidade e na distribuição do sistema radicular, após todos os anos de aplicações consecutivas.

Possivelmente, fatores inerentes ao solo do experimento, que é de natureza argilosa, tais como teor médio de matéria orgânica ao redor de $1,6 \%$, tenham contribuído para a manutenção dos íons herbicidas à profundidades bem pequenas, proporcionando um bom controle das sementes germinantes sem entretanto, atingir um maior volume destas radicelas superficiais, caracterizando uma perfeita seletividade topônica. Tais fatores são citados por Hance (5), como importantes na relação herbicida solo.

Victória Filho et al. (19), através de dados levantados anteriormente na mesma área experimental do presente trabalho, observaram que não houve influência dos herbicidas nas propriedades químicas do solo e que tais produtos químicos também não proporcionaram alterações maléficas nas propriedades físicas, avaliadas através da densidade aparente, permeabilidade e estruturação, com resultados mais adequados que os obtidos no solo sob cultivo convencional (gradagem).

Outros experimentos relaciona dos com os efeitos dos herbicidas no sistema radicular de laranieiras iá foram realizados por outros 
Quadro 2. Peso médio ( $\mathrm{cg}$ ) das radicelas das laranjeiras, nas diferentes distâncias do tronco, profundidade do solo e direções de amostragem.

\begin{tabular}{|c|c|c|c|c|c|c|c|c|}
\hline \multirow{3}{*}{ NQ } & \multirow{3}{*}{ Tratamentos } & \multirow{3}{*}{$\begin{array}{l}\text { Profun- } \\
\text { didade } \\
(\mathrm{cm})\end{array}$} & \multicolumn{6}{|c|}{ Distância do tronco $(\mathrm{cm})$} \\
\hline & & & \multicolumn{2}{|c|}{80} & \multicolumn{2}{|c|}{160} & \multicolumn{2}{|c|}{240} \\
\hline & & & A & B & A & B & A & B \\
\hline \multirow{2}{*}{\multicolumn{2}{|c|}{ 1. Testemunha capinada }} & $0-15$ & $62,67 *$ & 31,33 & 21,00 & 12,33 & 16,33 & 17,67 \\
\hline & & $15-30$ & 32,67 & 10,33 & 10,67 & 12,67 & 18,67 & 10,67 \\
\hline \multirow{2}{*}{\multicolumn{2}{|c|}{ 2. Fluometuron }} & $0-15$ & 46,33 & 75,00 & 26,67 & 24,33 & 15,33 & 16,00 \\
\hline & & $15-30$ & 20,33 & 15,33 & 8,00 & 7,67 & 20,00 & 15,33 \\
\hline \multirow{2}{*}{\multicolumn{2}{|c|}{ 3. Simazine }} & $0-15$ & 68,00 & 75,33 & 35,33 & 43,33 & 20,33 & 27,00 \\
\hline & & $15-30$ & 17,67 & 18,33 & 6,67 & 16,33 & 5,33 & 16,00 \\
\hline \multirow{2}{*}{\multicolumn{2}{|c|}{ 4. Atrazine }} & $0-15$ & 52,00 & 35,67 & 28,67 & 60,33 & 14,67 & 12,00 \\
\hline & & $15-30$ & 28,00 & 11,67 & 5,33 & 9,00 & 9,33 & 11,33 \\
\hline \multirow{2}{*}{\multicolumn{2}{|c|}{ 5. Bromacil }} & $0-15$ & 38,67 & 47,00 & 27,33 & 6,33 & 21,33 & 20,33 \\
\hline & & $15-30$ & 12,33 & 16,33 & 13,67 & 10,00 & 10,33 & 9,00 \\
\hline \multirow{2}{*}{\multicolumn{2}{|c|}{ 6. Bromacil $(40 \%)+$ diuron $(40 \%)$}} & $0-15$ & 63,33 & 44,67 & 41,33 & 28,00 & 15,33 & 16,33 \\
\hline & & $15-30$ & 34,00 & 13,00 & 31,00 & 5,33 & 8,00 & 14,33 \\
\hline \multirow{2}{*}{\multicolumn{2}{|c|}{ 7. Bromacil $(53,3 \%)+$ diuron $(26,7 \%)$}} & $0-15$ & 75,33 & 37,67 & 37,67 & 33,33 & 11,33 & 23,33 \\
\hline & & $15-30$ & 14,33 & 16,67 & 6,67 & 22,67 & 10,67 & 15,00 \\
\hline \multirow{2}{*}{\multicolumn{2}{|c|}{ 8. Terbacil }} & $0-15$ & 62,00 & 65,67 & 29,33 & 12,67 & 13,67 & 14,33 \\
\hline & & $15-30$ & 37,00 & 33,67 & 13,00 & 8,67 & 9,00 & 11,33 \\
\hline \multirow{2}{*}{\multicolumn{2}{|c|}{ 9. Oxadiazon }} & $0-15$ & 47,33 & 24,00 & 41,00 & 41,00 & 24,00 & 29,67 \\
\hline & & $15-30$ & 19,00 & 6,67 & 25,33 & 6,67 & 13,33 & 12,67 \\
\hline \multirow{2}{*}{\multicolumn{2}{|c|}{ 10. Dichlobenil }} & $0-15$ & 22,00 & 58,00 & 39,67 & 69,00 & 42,67 & 15,67 \\
\hline & & $15-30$ & 12,00 & 10,67 & 25,67 & 19,67 & 16,33 & 27,00 \\
\hline \multirow{2}{*}{\multicolumn{2}{|c|}{ 11. Dichlobenil }} & $0-15$ & 32,67 & 52,67 & 7,33 & 25,33 & 33,67 & 54,67 \\
\hline & & $15-30$ & 25,33 & 16,33 & 6,33 & 17,67 & 22,67 & 15,33 \\
\hline
\end{tabular}

$A=$ amostra coletada entrelinhas; $B=$ amostra coletada entreplantas

* média de três repetiçoes. 
Quadro 3. Análise de variância dos pesos de radicelas obtidos, para os diferentes fatores avalia dos e suas interações, nas amostras obtidas, segundo o esquema fatorial $11 \times 2 \times 3 \times 2$.

\begin{tabular}{|c|c|c|}
\hline Causas de Variação & G.L. & $\mathrm{F}$ \\
\hline Tratamento (T) & 10 & $0,55 \mathrm{~ns}$ \\
\hline Profundidade (P) & 1 & $64,28 * *$ \\
\hline Distância (D) & 2 & $15,86 * *$ \\
\hline Direção (Di) & 1 & $0,05 \mathrm{~ns}$ \\
\hline $\mathrm{T} \times \mathrm{P}$ & 10 & $0,40 \mathrm{~ns}$ \\
\hline $\mathrm{T} \times \mathrm{D}$ & 20 & $1,13 \mathrm{~ns}$ \\
\hline $\mathrm{T} \times \mathrm{Di}$ & 10 & $0,84 \mathrm{~ns}$ \\
\hline $\mathrm{P} \times \mathrm{D}$ & 2 & $8,67 * *$ \\
\hline $\mathrm{P} \times \mathrm{Di}$ & 1 & $0,38 \mathrm{~ns}$ \\
\hline $\mathrm{D} \times \mathrm{Di}$ & 2 & $0,43 \mathrm{~ns}$ \\
\hline$T \times P \times D$ & 20 & $0,50 \mathrm{~ns}$ \\
\hline $\mathrm{T} \times \mathrm{P} \mathrm{d} \mathrm{Di}$ & 10 & $0,42 \mathrm{~ns}$ \\
\hline $\mathrm{T} \times \mathrm{D} \times \mathrm{Di}$ & 20 & $0,62 \mathrm{~ns}$ \\
\hline$P \times D \times D i$ & 2 & $0,05 \mathrm{~ns}$ \\
\hline $\mathrm{T} \times \mathrm{P} \times \mathrm{D} \times \mathrm{Di}$ & 20 & $0,50 \mathrm{~ns}$ \\
\hline (Tratamentos) & 131 & - \\
\hline Blocos & 2 & 3,66 \\
\hline Resíduo & 262 & - \\
\hline TOTAL & 395 & - \\
\hline
\end{tabular}


pesquisadores. Kimbal et al.(1951), citados por Moreira (13), verificaram que a gradagem proporcionou cortes na maior parte das raízes superficiais e que o controle químico das plantas daninhas favoreceu o crescimen to de radicelas na superfície do solo. Jordan (7) relata que arvores de pomares trata dos com herbicidas, são mais sadias e vigorosas do que as de roça das ou gradadas, isto devido à redução da competição pela luz,água, espaço e nutrientes, além da dimi nuição dos cortes de radicelas pelos implementos mecânicos. Marti nez et al. (11) estudaram a influência de nove praticas de cultivo do solo, sobre o sistema radicular de laranjeiras 'Hamlin', incluindo um trat amento em que se efetu ou a aplic ação de herbicidas e concluíram que não houve diferença signific ativa entre eles .

Resultados discordantes aos obtidos neste trabalho, foram observados por Moreira (13) que, ao com parar a quantidade de radicelas da faixa tratada com herbicid as em relação as das entrelinha gradada, na profundidade de 0-15 cm, verificou a influência negativa de alguns dos produtos químicos.

Comparando-se as medias dos pesos das radice las nas profundidades de $0-15 \mathrm{~cm}$ e 15 a $30 \mathrm{~cm}$, pelo teste de Tukey (Quadro 4), visualiza-se uma quantidade bem maior na camada mais superficial, superior ao dobro das encontradas na camada mais profunda. As diferentes distancias do tronco também foram comparadas e houve diferença significativa da distancia de $80 \mathrm{~cm}$ do tronco com as outras duas (160 e $240 \mathrm{~cm})$, que por sua vez não diferiram entre si. A direção parece não influenciar significativamente na quantidade de raízes do cultivar Natal, sobre porta-enxerto de limão 'Cravo', se o espaça mento for mais ou menos igual para as plantas dentro das linhas e entre-linhas. Este fato pode ser levado em consideração no estabelecimento do espaçamento a ser utilizado quando do plantio das mudas no campo e na localização do adubo aplicado, no pomar forma do.

No estudo das interações, conforme aparece no Quadro 3, a análise estatística também mostrou, de forma coerente, interação altamente significativa entre profundidade (P) e distância (D). Feito -odesdobramento dos graus de liberda de dessa interação (Quadro 5), observou-se que as distancias diferiram apenas na profundidade de 0 a $15 \mathrm{~cm}$, e por sua vez, as profundidades também apresentatam tal variação significativa, nas distancias de 80 e $160 \mathrm{~cm}$, a partir do tronco. O teste de Tukey retificou o efeito da distancia de amostragem na variação das médias dos pesos das radicelas, somente na pro fundidade de 0 a $15 \mathrm{~cm}$ (Quadro 6). Esse fato foi considerado normal, visto que ness a camada do solo $(015 \mathrm{~cm})$, situa-se a maior parte das radicelas, que diminuem acentuadamente a medida que vão afas tando do tronco. Já para a profundidade de 15 a $30 \mathrm{~cm}$, tal fato não ocorreu pois a quantidade de radicelas era inferior, distribuindo-se uniformemente nas diferentes distancias do tronco.

As Figuras 1 e 2 apresentam os dados expressos em porcentagem, referentes às distribuições horizontal e vertical das radicelas, respectivamente, nos diferentes trata mentos. Pela Figura 1, observa-se que na camada superficial (0 a $15 \mathrm{~cm}$ ), aproximadamente $80 \%$ das radicelas localizam-se até a distân- 
Quadro 4. Comparação dos pesos médios ( $\mathrm{cg}$ ) das quantidades de radicelas entre os fatores de amos tragem, avaliados através do Teste de Tukey.

\begin{tabular}{ll}
\hline Metodologia para obtenção das amostras & Médias (*) \\
\hline - Distância (cm) & 34,40 a \\
$\quad 80$ & 22,65 b \\
160 & 17,82 b \\
240 & \\
- Profundidade (cm) & 34,86 a \\
0 a 15 & 15,05 b \\
15 a 30 & \\
- Direção & 25,23 a \\
entrelinha & 24,69 a \\
entreplanta
\end{tabular}

d.m.s. distância a $5 \%=7,15$

d.m.s. profundidade a $5 \%=4,87$

d.m.s. direção a $5 \%=4,87$

(*) médias seguidas de pelo menos uma letra igual, não difere de forma estatisticamente significativa ao nivel de $5 \%$ de probabilidade, segundo o teste de Tukey. 
Quadro 5. Anālise de variância com desdobramentos dos graus de liberdade, da interação Distância (D) $x$ Profundidade (P). 
Quadro 6. Comparação dos pesos médios $(\mathrm{cg})$ das quantidades de radicelas referentes à interação Profundidade (P) x Distância (D), através do teste de Tukey.

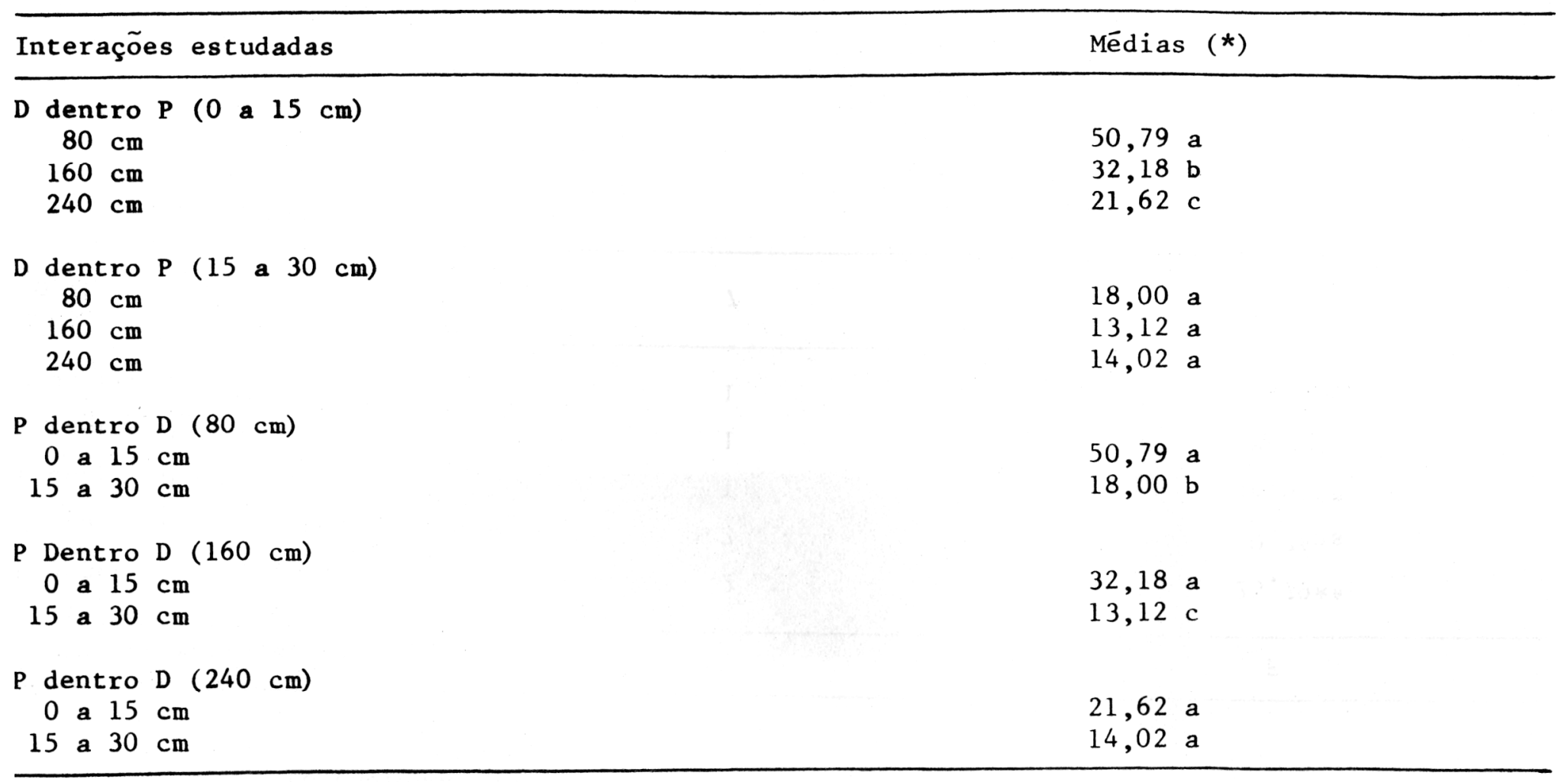

d.m.s. D dentro $P$ a $5 \%=10,11$

d.m.s. P dentro D a $5 \%=8,43$

(*) médias seguidas de pelo menos uma letra igual, não diferem de forma estatisticamente signifi cativa ao nível de $5 \%$ de probabilidade, segundo o teste de Tukey. 

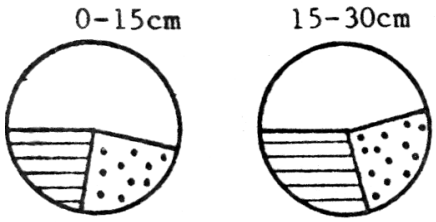

Testemunha capinada
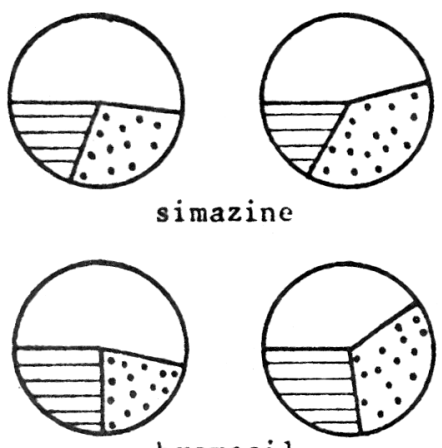

bromacil

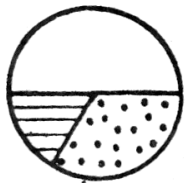

bromacil $(53,3 \%)+$ diuron $(26,7 \%)$
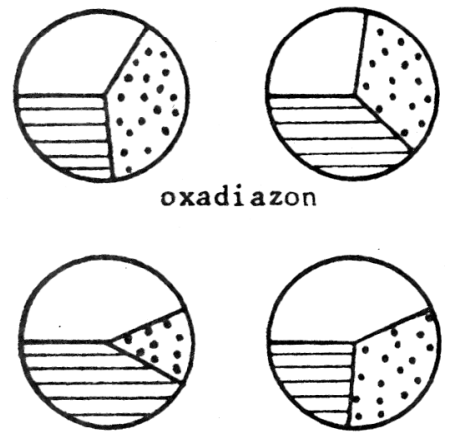

dirhlobenil

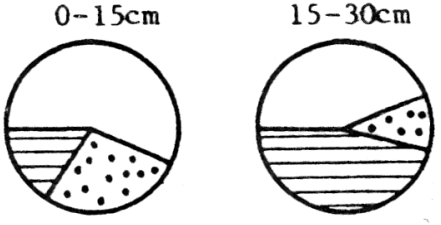

f luometuron

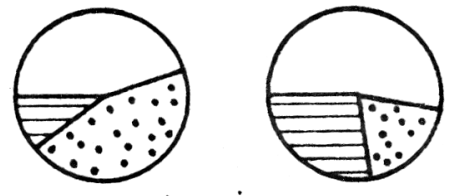

atrazine

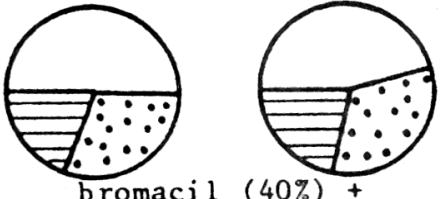

diuron $(40 \%)$
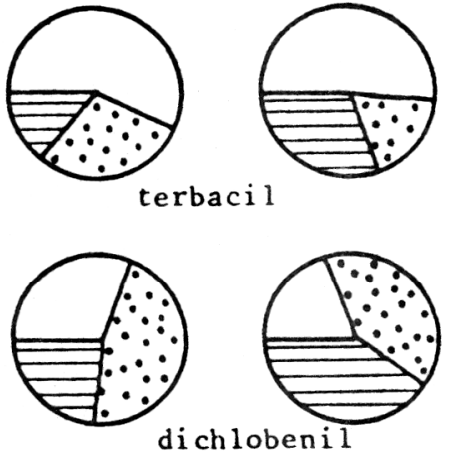

$\square 80 \mathrm{~cm}$

DISTÂNCIA DO

$\therefore 160 \mathrm{~cm}$

TRONCO

Figur a 1. Quantidades de radic elas, expressas em porcentagem do total existente em cada profundidade, nas diferentes distâncias do tronco - Distribuição horizontal. 

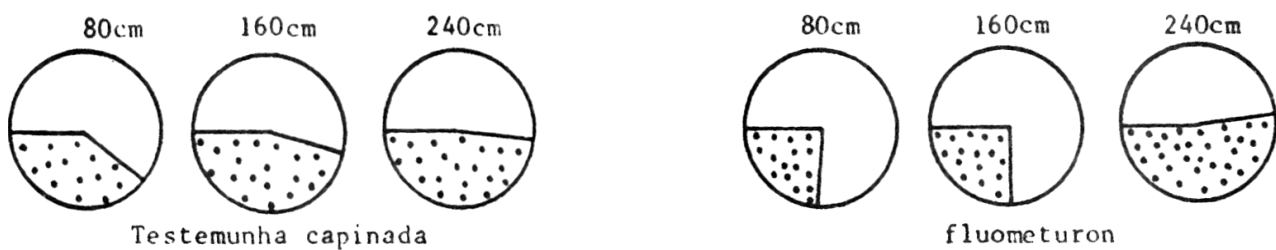

fluometuron
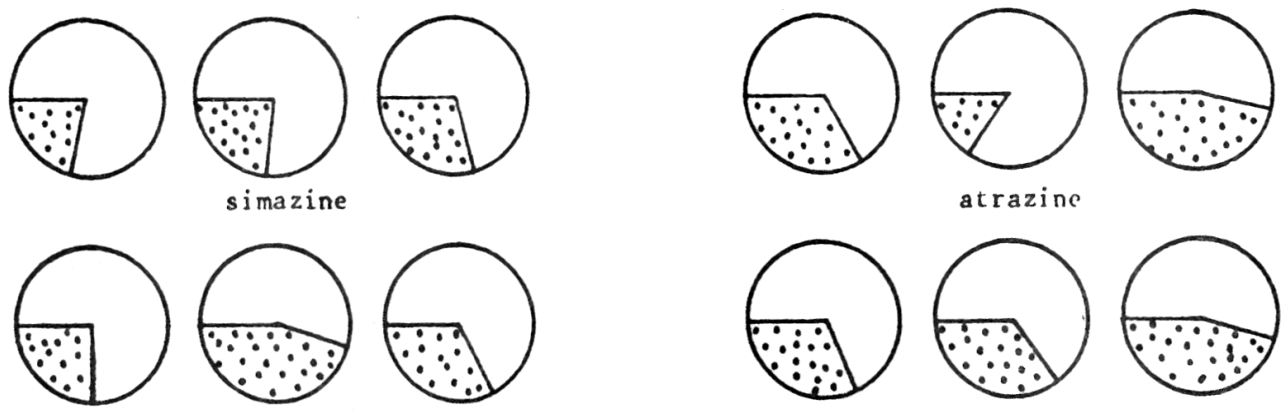

bromacil (40\%) + diuron (40\%)
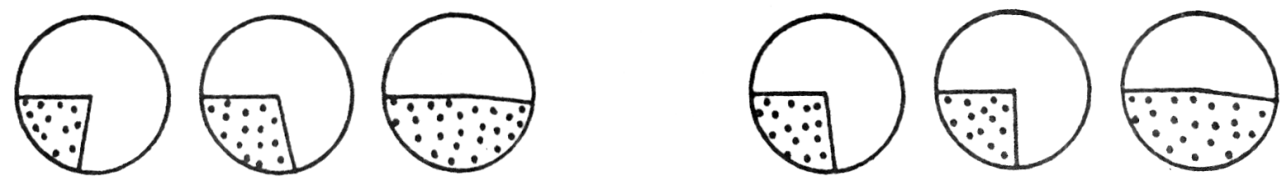

bromacil $(53,3 \%)+$ diuron $(26,7 \%)$
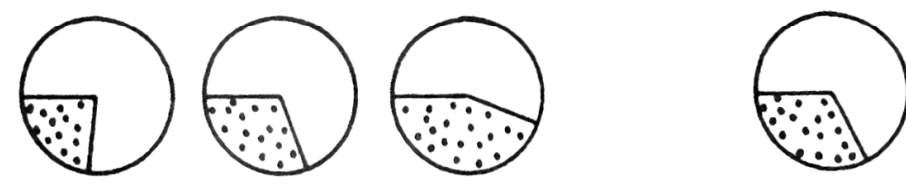

terbacil

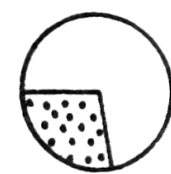

dichlobenil
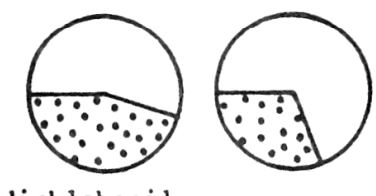

$\square 0-15 \mathrm{~cm}$

3.9. $15-30 \mathrm{~cm}$

Figura 2. Quantidades de radic elas, expressas em porcentagem do total existente em cada distância, nas diferentes profundidades do solo - Distribuição vertical. 
cia de $160 \mathrm{~cm}$ do tronco. Na cama da imediatamente inferior, cerca de $70 \%$ estavam presentes nesta mesma distância. Verifica-se também que, pela Figura 2, existem aproximadamente $75 \%, 70 \%$ e $60 \%$ de radicelas, para as distâncias de 80 , 160 e $240 \mathrm{~cm}$ do tronco, respectivamen te.

Os resultados obtid os suge rem que as práticas de adubação, irri gação, gradagem e aplicação de capinas mecânicas, quando realizadas em pomares deste cultivar, devem considerar a existência de maior quantidade de radicelas na camada superficial do solo $\left(\begin{array}{llll}0 & \text { a } & 15 & \mathrm{~cm}\end{array}\right)$ e à distancias não superiores a $160 \mathrm{~cm}$.

Os herbicidas residuais, desde que aplicados de forma correta e em doses adequadas, não causam danos de quaisquer natureza ao sistema radicular, porém é aconselhável restringi-los a uma faixa ao longo das linhas, mantendo-se uma cobertura morta ou de plantas roça das na entre-linha, principalmente em áreas de maior declividade e com altos índices pluviométricos,o que auxilia sobremaneira no controle da erosão.

\section{LITERATURA CITADA}

1. Caetano, A.A. Tratos culturais. In: Rodrigues, 0 . \& Viegas, F. coord. Citricultura Brasileira. Campinas, Fundação Cargil1, 1980. v. 2., p.42-466.

2. Durigan, J.C. Herbicidas e plantas daninhas em citrus. Jaboticabal, FCAVJ, 1977. 37p. (Revisão bibliográfica).

3. Ford, H.W. The influence of rootstock and tree age on root distribution of citrus. Proceedings American Soc. Horticultural Sci, 62: 137-142, 1954.

4. Goren, R. \& Monselise, S.P.Stu dies on the utilization of herbicides in citrus orchards in Israel as related to efficiency. In: International Cytrus Symposium, 19, Riverside, 1969. Proceedings, vol. 1, p.483492.

5. Hance, R.J. Herbicide persistence and breakdown in soil in the long term. Annals of Applied Biology, 91: 129$131,1979$.

6. Herdholdt, J.A. Weed control in South Africa Citrus orchards. In: Internationa1 Citrus Symposium, 19, River side, 1969. Proceedings. vol. 1, p.459-502.

7. Jordan, L.S. Benefits and pro blems of herbicides use in citriculture. Internatio nal Society Citriculture, $\mathrm{R} \overline{\mathbf{i}}$ verside, 1978. Proceedings. p. 209-214.

8. Jordan, L.S. \& Day, B.E. Weed control in citrus. In: Reuther, W.; Batchelor, L.D.; Webber, H.D. ed. The Citrus Industry, California University of California, 1973. vol. 3, p.82-87.

9. Jordan, L.S.; Day, B.E.; Ruse11, R.C. Herbicides in citrus trees and soils. In: International Citrus Symposium, 19, Riverside, 1969. Proceedings, vol. 1, p.463466.

0. Leydon, R.F. Development of the herbicide program in Te xas. In: International $\mathrm{Ci}=$ trus Symposium, 19, Riverside, 1969. Proceedings. p. $473-477$. 
11. Martinez, A.A.; Rodrigues, 0.; Inforzato, R.; Abramides, S.E. Influência de nove práticas de cultivo do solo no sistema radicular de laranjeiras Haml in enxertadas em laranjas-caipira. In: Reu nião Anual da Sociedade Brá sileira para o Progresso da Ciência, 22\%, Salvador, BA, 1970. Resumos. p.221.

12. Montenegro, H.W.S. Contribuição ao estudo do sistema radicular das plantas cítri cas. Piracicaba, ESALQ7 USP, 1960. 143p. (Tese de Cátedra).

13. Moreira, C.S. Estudo da distribuição do sistema radicu lar da laranjeira Pera ( $\mathrm{C} i-$ trus sinensis L.) Osbeck.), com diferentes manejos de solo. Piracicaba, ESALQ/ USP, 1983. 97p. (Tese de Livre-docência).

14. Oohata, J.T. Herbicides in Japanese citrus growing. Internationa1 Cytrus Symposium, 19, Riverside, 1969. Proceedings, vol. 1, p.493 -497 .

15. Reuther, W. Cultural Practi ces -75 years of citrus re search. California Agricul tura, 36(11-12): 1-16,1982. 16. Ryan, G.F. The use of chemical for weed control in Flo rida citrus. In: Interna tional Citrus Symposium, 19, Riversidade, 1969. vol. 1, p.467.472.

17. Torrise, S. Development and use of herbicides in Sicilian citrus orchard as a means for nontillage weed control. Internationa1 $\mathrm{Ci}$ trus Symposium, 19, Riverside, 1969. vol. I, p.479482.

18. Victória Fo, R.; Corsini, P. C.; Pitelli, R.A. Efeitos do uso contínuo de herbici das em citros (Citrus sinensis L. Osbeck.). II Efeitos no desenvolvimento, nos teores de macro e micronutrientes e nas propriedades físicas e químicas do solo. In: CONGRESSO BRASILEIRO DE HERBICIDAS E PLANTAS DANINHAS, 15\%; CONGRESSO DE LA ASOCIACION LATINOAMERICANA DE MALEZAS, 79, Belo Horizonte, 1984. Resumos. p.151-152. 